\title{
Simpler semantics for computational and cognitive linguistics
}

\author{
Hywel Evans \\ TSURU UNIVERSITY \\ evans@tsuru.ac.jp
}

\begin{abstract}
Certain consequences are considered regarding a simpler, more cognitively plausible treatment of semantics in SignBased Construction Grammar, a cognitive, unificationbased theory of language. It is proposed that a construction grammar may be able to improve its coverage of core linguistic phenomena in line with minimalist goals (Chomsky 1993). Suggestions are offered regarding relative clauses and wh-expressions to show that a more straightforward account is available, one that allows a unified treatment of scope for quantifiers and wh-expressions.
\end{abstract}

\section{Introduction}

A review is presented regarding convincing evidence relating to a special morphology for the highest verb in relative clauses in Korean (Sag 1997). This suggests a straightforward formal treatment of a number of phenomena, including floating quantifiers (Yoo 2002), that utilizes a blend of Minimal Recursion Semantics (Copestake et al. 2005) and Frame Semantics (Fillmore 1982). In particular, it is noted that conditions on amalgamation (inheritance of features from more than one source) of frames from daughters to mother phrases (while suggesting that the mother inheriting features from daughters metaphor is clearly not necessarily the most natural one available) allows us to deal with certain complications regarding the inheritance of semantic features in unification-based approaches (e.g. Pollard \& Sag 1994). This kind of approach can also be very naturally and simply extended to cover data from Modern Standard Arabic (Alqurashi \& Borsley 2012).

These simplified mechanisms may also be applied in order to provide a unified treatment of $w h$-phrases and quantifiers, following Pollard \& Yoo (1998). This allows a number of simplifications. In particular, it will be shown that the invocation of a special semantic $w h$-feature, involving the unnecessary rejection of a unified treatment of scope, introduces serious 
difficulties in Sign-Based Construction Grammar (SBCG) that do not arise under a unified approach.

\subsection{Cognitive underpinnings}

In Head-Driven Phrase Structure Grammar (HPSG), conditions on inheritance of semantic features have traditionally been startlingly not head-driven, with only adjunct daughters contributing semantic features to mother phrases in head-adjunct structures. From a cognitive perspective, this is clearly anti-intuitive (not just because having mothers inherit from daughters seems odd), with adjuncts carrying the semantic content of the phrases they select. Having adjectives carry the semantic features of selected nominals, for example, may be unproblematic from a formal, computational perspective but seems to sit less well with linguistic proposals that are expected to conform to a cognitive view of language use (Tomasello 2003) and language change (Croft 2000).

The need for a cognitively plausible account is intensified with the development of SBCG (Boas \& Sag 2012), a cognitive theory of signs as mediating the correspondence between form and meaning. For example, relative clauses that select nominals are not expected to be semantically distinct from non-relative clauses. Floating quantifiers that look like ordinary determiners are not expected to carry clausal content (Yoo 2002). As SBCG employs a blend of Minimal Recursion Semantics (MRS) and Frame Semantics, content is amalgamated (inherited from multiple sources) across daughters, allowing considerable simplification. However, this has gone largely without comment in the literature, and a number of unnecessary complications remain.

I make a number of suggestions regarding a minimalist approach to SBCG. In particular, I suggest a unified FRAMES-based treatment of scope for wh-expressions and quantifiers. It is argued that the inventory of features can be radically reduced while extending the theory's coverage of core linguistic phenomena in a more cognitively plausible manner.

\section{Relative clauses}

Discussion regarding relative clauses (RC) in HPSG has centered on the use of phonologically empty complementizers. While Pollard and Sag (PS 1994) made extensive use of null complementizers, Sag (1997) observed that many languages (Korean and Bantu, for example) show special morphology on the highest verb in a relative clause. So we get the contrast between (1) and (2), taken from Sag (1997):

(1) John-i chayk-ul ku sangca-ey neh-ess-ta. John-NOM book-ACC that box-LOC put-PAST-DECL 'John put the book in the box.'

(2) [[John-i chayk-ul neh-un] sangca-ka] khu-ta. John-NOM book-ACC put-REL box-NOM big-DECL 'The box in which John put the book is big.' 
Verbs carrying the [MOD N'] feature might be expected to carry the relevant morphology, as can be seen from the illustration (taken from Sag (1997) below in (3).

(3) Relative clause specifications in Korean:

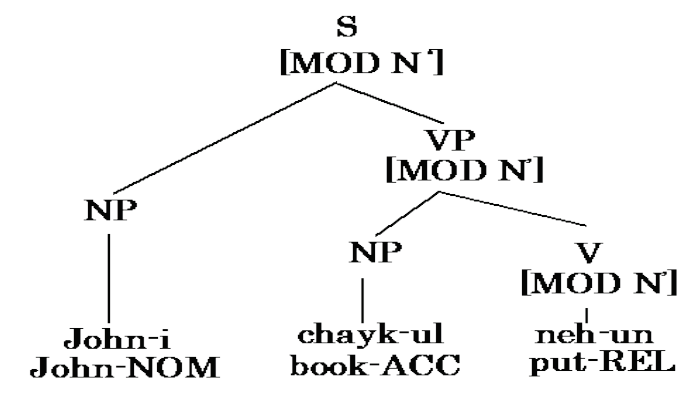

Since PS (1994), semantic features have traditionally been identified with a single daughter, with structure-sharing between the daughter and the mother. In a head-adjunct structure, this single daughter is the adjunct. In this approach, if the S in (3) modifies a nominal structure of some sort, it would then be required to carry the semantic features of the nominal as well as its own, necessitating an anomalous treatment of CONTENT (CONT) for relative clauses. In fact, Sag (1997) thought this was sufficiently strange to warrant a special head-relative-phrase constraint to ensure that verbs in RCs do not actually have special CONT values after all. More recently, Alqurashi and Borsley (AB 2012) object to such an ad hoc arrangement and prefer the use of null complementizers.

A natural solution is to assume that the semantic features of phrases are not determined exclusively via inheritance from a single (semantic-) head daughter. This kind of solution actually offers itself in line with Boas and Sag's (2012) overview of SBCG, although it has attracted little direct attention. In MRS, content is amalgamated across daughters (Purver \& Ginzburg 2003: 343) rather than inherited from a single daughter, so the problem of anomalous RC content disappears.

\subsection{Arabic data}

Even though a straightforward alternative is available, Alqurashi and Borsley (AB, 2012) favor a return to the classic HPSG (PS 1994) approach in order to handle data related to Modern Standard Arabic. Arabic RCs may only function without special (phonologically overt) complementizers in conjunction with indefinite nominals. Hence, we see the following contrasts (gaps indicated with underscores):
(4) jaa?a
l-walad-u
[llaði
qaabaa
l-malika] came.3.M.SG DEF-boy-NOM that met.3.M.SG
DEF-king-ACC
'The boy who met the king came' 


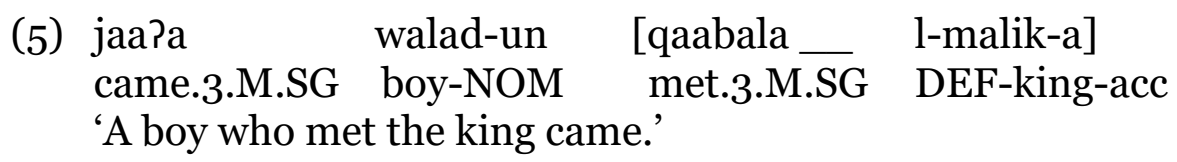

$\mathrm{AB}$ (2012) note a problem with Sag's treatment of head-relative-phrase of the sort indicated in (3). In particular, it does not generalize to constructions with overt complementizers. Indeed, we might expect MOD NP verbs in Arabic to carry the same kind of specifications as special complementizers such as llaði in (4). As stated earlier, this requires the clause to carry a complex 'nominal' CONT. To get around this problem, AB (2012) posit a phonetically null complementizer mediating between a clausal complement and modified nominal in (5). This allows the complement S to simply carry its normal propositional CONT.

This null-complementizer approach appears to handle the data adequately. However, Sag's (1997) evidence suggesting special morphology for verbs in RCs goes without an explanation because verbs no longer carry a MOD feature. Also, AB's (2012) null-complementizer solution is basically no less ad hoc than Sag's head-relative-phrase constraint. A simpler, and more cognitively plausible, solution would be to assume that the semantic features of phrases are not determined exclusively via inheritance from a single head daughter. Rather, semantic features are structure-shared (inherited) between mothers and head daughters with the exception that FRAMES are amalgamated across daughters. Importantly, this kind of solution actually naturally offers itself in line with Boas and Sag's (2012) overview of Sign-Based Construction Grammar (SBCG), although this has not been stated explicitly anywhere as far as I can tell.

\subsection{Frame amalgamation, storage, and scope}

First, consider conditions on amalgamation of FRAMES and the manner in which scope is determined.

(6) Syntactic and semantic features of book in SBCG:

$$
\left[\begin{array}{l}
\text { fn-lxm } \\
\text { FORM }<\text { book }> \\
\text { SYN }\left[\begin{array}{l}
\text { CAT [noun] } \\
\text { VAL }<\text { DetP }>
\end{array}\right] \\
\text { SEM }\left[\begin{array}{l}
\text { INDEX } i \\
\text { FRAMES }<\left[\begin{array}{l}
\text { book- } f r \\
\text { LABEL } I_{o} \\
\text { ENTITY } i
\end{array}\right]>
\end{array}\right]
\end{array}\right.
$$

In SBCG, a common noun lexeme such as book, shown above, needs a determiner in order to satisfy its syntactic VALENCY (VAL) requirements. 
Semantic features include an INDEX and FRAMES, the latter based on 'Frame Semantics' (Fillmore 1982, 1985; Fillmore and Baker 2010). In SBCG, this is blended with Minimal Recursion Semantics (MRS). As suggested, in MRS approaches (Copestake et al. 2005) to semantic analysis, it is assumed that FRAMES are amalgamated across daughters by default, rather than being inherited from a single daughter. Of course, this can also be understood in a common sense manner in terms of inheritance, such that the syntactic and semantic features of a phrase are inherited from the head daughter, except that the FRAMES will be inherited from all daughters. It simply means that the grammar is somewhat less headdriven, with conditions on inheritance somewhat different for syntactic and semantic features.

One might also presume that a determiner such as every will have some way of selecting a nominal, in this case via the SELECT feature (MOD in HPSG). This allows the determiner's INDEX and bound variable (BV) feature in FRAMES to be identified with the selected N'.

(7) Syntactic and semantic features of every in SBCG:

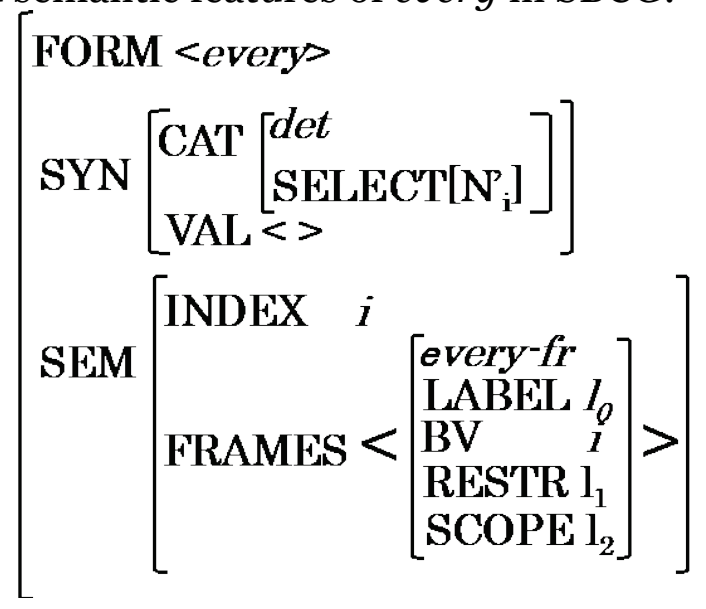

SEM features of a noun phrase such as every book may therefore be represented as in (8).

(8) Partial feature representation for every book:

$$
\left[\begin{array}{l}
\text { FORM < every, book> } \\
\text { SEM } \left.\left[\begin{array}{l}
\text { sem-obj } \\
\text { INDEX } i \\
\text { FRAMES } \left.<\left[\begin{array}{l}
\text { every-fr } \\
\text { LABEL } I_{1} \\
\text { BV } i \\
\text { RESTR } 1_{2} \\
\text { SCOPE } 1_{3}
\end{array}\right],\left[\begin{array}{l}
\text { book-fr } \\
\text { LABEL } \\
\text { ENTITY } I_{2}
\end{array}\right]>\right]
\end{array}\right]\right]
\end{array}\right.
$$


In this approach, both the syntactic and semantic features of a phrase are straightforwardly identified with the syntactic head, in this case book. However, FRAMES are inherited from all daughters as VALENCY features are naturally checked off. In an NP such as every book, the LABEL of the book-fr is identified with the RESTR value of the every-fr. The LABEL and SCOPE values of the every-fr are not yet identified with any other frame, meaning that the scope has not yet been determined. In addition to INDEX and FRAMES, a third semantic feature, LTOP (LOCAL-TOP), omitted here, plays a part in scope assignment in indicating the 'top' frame in a sentential structure, for example. This pattern of underspecification is satisfactorily in line with previous treatments of quantifiers, in accordance with Cooper's $(1975,1983)$ rule of storage. In a satisfactorily metaphorical sense, this inheritance mechanism allows us to preserve the analogy that quantifiers are stored and passed up to higher levels of structure until appropriate conditions on scope may be assigned. Scope can be determined without any extra requirement for special inheritance conditions. Also, as adjuncts are not required to carry the semantic information of heads, an SBCG account that utilizes FRAMES and MRS does not suffer from an anomalous semantics for modifiers. I will argue that this cognitively plausible simplification of the semantics sets off a cascade of consequences that are extremely important for minimalist conceptions of linguistic theory. In particular, I will argue for a unified, minimalist FRAMES-based treatment of $w h$-expressions in SBCG.

AB's (2012) Arabic data can be handled straightforwardly if we assume that verbs bearing a MOD (SELECT is the notational variant in SBCG) feature carry certain specifications.

(9) Specifications borne by SELECT NP verb-forms:

$$
\left[\begin{array}{l}
\operatorname{SYN}\left[\begin{array}{l}
\operatorname{CAT}\left[\begin{array}{l}
\text { verb } \\
\mathrm{SELECT} \mathrm{NP}_{i}[-d e f]
\end{array}\right] \\
\mathrm{VAL}<> \\
\mathrm{GAP}<\mathrm{NP}_{i}>
\end{array}\right]
\end{array}\right]
$$

A verb in Arabic may only SELECT an NP in case it is not marked definite and has its INDEX shared with the GAP value carried by the verb. This will handle examples such as (5). The verb will contribute its FRAMES as usual, and these will be amalgamated onto the NP CONT, as expected. The CONT of overt complementizers, then, will naturally be identical to the CONT of the S complement. In a relative clause head-adjunct NP with NP head and $\mathrm{CP}$ adjunct, the CONT will be structure-shared with the head NP and FRAMES will be appropriately amalgamated. 
(10) Specifications borne by the complementizer Pallađi:

$$
\left[\operatorname{SYN}\left[\begin{array}{l}
\text { CAT }\left[\begin{array}{l}
c \\
\text { SELECT NP }
\end{array}:\left[\begin{array}{l}
\mathrm{NUM} \text { sing } \\
\text { GEND masc }
\end{array}\right]\right. \\
\mathrm{VAL}<\mathrm{S}\left[\mathrm{GAP}<\mathrm{NP}_{1}>\mathrm{l}>\right.
\end{array}\right]\right.
$$

Assuming a general rule that complementizers take NPs marked definite, ?allaði will be required to take a singular, masculine NP unspecified for case. This NP will be structure-shared with the GAP in the sentential complement. Other complementizers differ minimally regarding the NP they select. For example, Pallati will be required for definite, singular, feminine NPs.

Assuming, with $A B$ (2012), that gaps and resumptive clitics (in bold below) may be treated as similar elements, we may also account for examples such as (11):
(11) jaa?a
l-waladaani
came.3.M.DUAL DEF-boy.DUAL.NOM
[llaðaani qaabala-humaa l-malik-u]
that met.3.M.SG-3.DUAL DEF-king-NOM
'The two boys whom the king met came.'

(12) Specifications for ?allaðaani:

$$
\left[\operatorname{SYN}\left[\begin{array}{l}
\operatorname{CAT}\left[\begin{array}{l}
c \\
\text { SELECT NP }[\text { nom }]: i
\end{array}\left[\begin{array}{l}
\text { NUM } t \text { wo } \\
\text { GEND masc }
\end{array}\right]\right. \\
\mathrm{VAL}<\mathrm{S}[\mathrm{GAP}<\mathrm{NP}>\mathrm{N}>
\end{array}\right]\right]
$$

In line with (12) we expect the complementizer to select NP[nom] but the GAP will only be structure-shared with the NUM and GEND features of the NP. Hence, the GAP features may be associated with a non-nominative argument, as in (11).

Crucially, SEM features need no special treatment. A complementizer may be assumed to have its INDEX structure-shared with that of the sentential complement. However, the complementizer's FRAMES list will naturally be empty. In a head-complement phrase headed by a complementizer, we expect the INDEX to be structure-shared with the head. FRAMES will be the amalgamation of the FRAMES of the daughters. Therefore, in a CP headed by a complementizer, we expect only the nonhead daughter to contribute FRAMES. 
(13) CP SEM features:

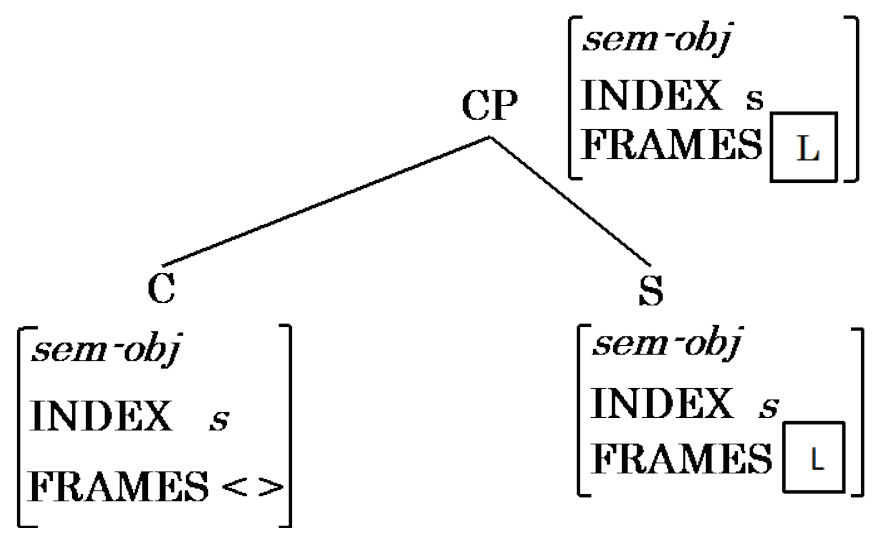

In a $\mathrm{CP}$ with complementizer head and sentential non-head daughter, the SEM features will be identical to the SEM features on the non-head daughter because the INDEX is inherited from the head daughter while the identity of FRAMES follows because the head-daughter FRAME list is empty.

(14) $\mathrm{NP}_{\mathrm{i}}$ with $\mathrm{CP}\left[\mathrm{SELECT} \mathrm{NP_{i }}\right]$ non-head daughter:

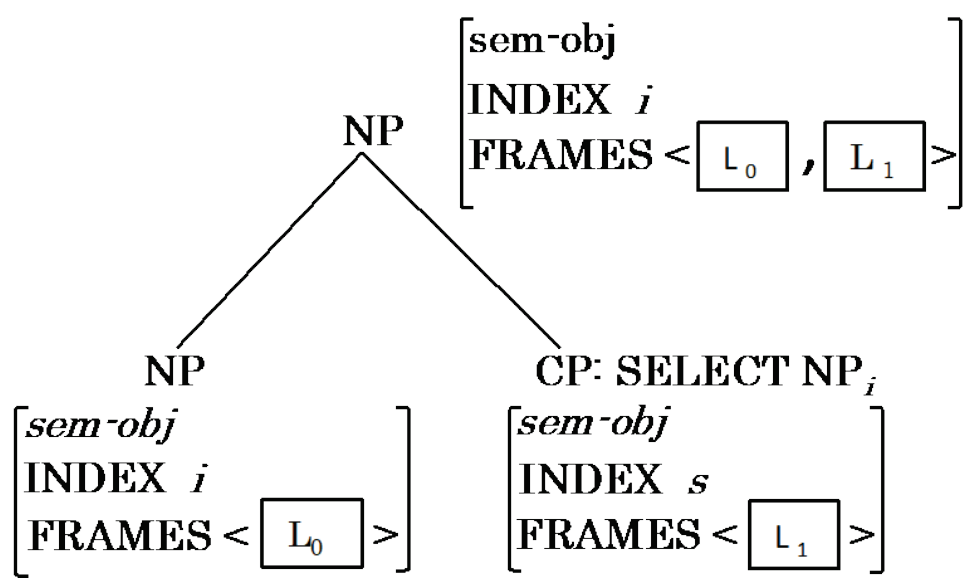

A head-adjunct construction with SELECT $<\mathrm{NP}>$ non-head daughter will take its INDEX (and LTOP) value from the head daughter, whether or not the non-head daughter is a CP or a bare MOD N' clause. The FRAMES of the daughters will, of course, be amalgamated on the mother phrase. There is no need to assume any special semantics for SELECT NP clauses.

In line with Sag (1997), wh-RCs in English will be required to carry a syntactic reflex REL feature. Conditions on inheritance of REL will be broadly parallel to conditions on inheritance of the syntactic reflex WH feature. 
(15) wh-rel-cl

$$
\left[\begin{array}{ll}
\text { HEAD } & \text { [MOD NP } \\
\text { NON-HD-DTRS } & <[\operatorname{REL}\{i\}>
\end{array}\right]
$$

This will be discussed further in the following section.

\subsection{Wh-expressions}

We have seen so far that conditions on inheritance of features follow a simple pattern whereby syntactic and semantic features are inherited from head daughters with the exception that FRAMES are amalgamated across daughters. Now consider the SBCG treatment of $w h$-expressions. Sag's (2012) constraints applying to filler-gap constructions are crucial in determining well-formed $w h$-sentences.

(16) Filler-head construction:

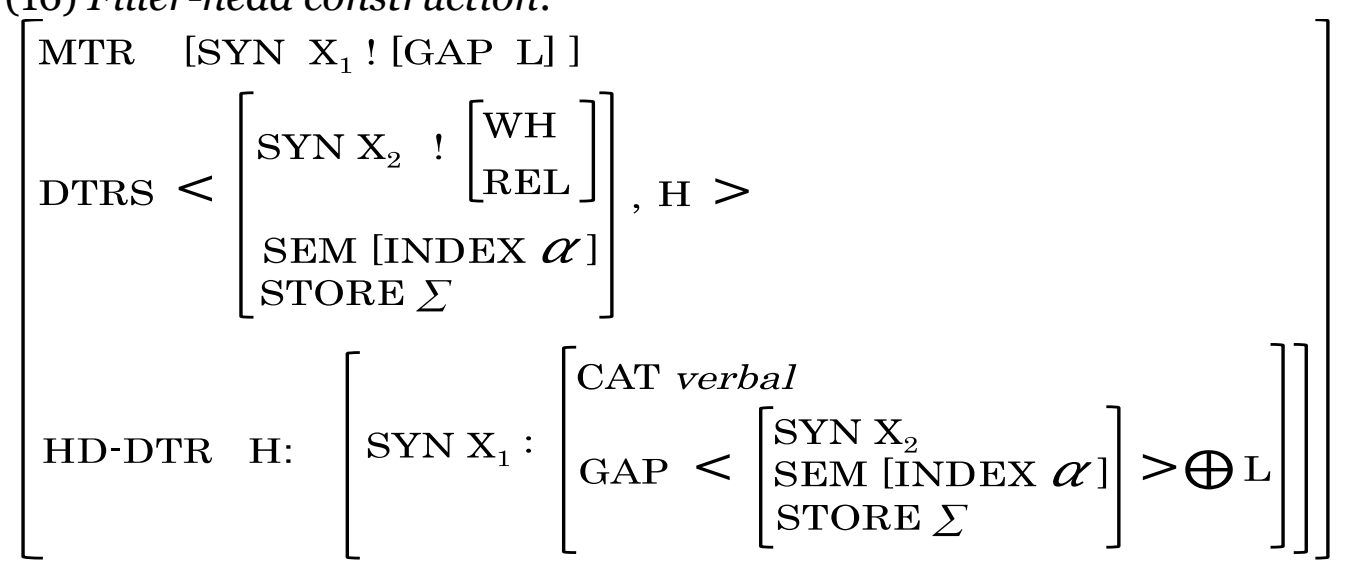

A filler-head construction will require the appropriate GAP value of the head daughter (HD-DTR), structure-shared with the syntactic features (SYN) of the filler (except for WH and REL features), to be removed from GAP values of the mother (MTR). WH and REL are the respective syntactic reflex features mentioned earlier. Of interest here, STORE is analogous to Pollard and Sag's (1994) treatment of quantificational expressions, based on Cooper's storage technique for dealing with scope ambiguities, also mentioned above. In this approach, all quantifiers start out in storage and are retrieved at the appropriate phrasal level in order to allow appropriate scope as LTOP, LABEL, and SCOPE values interact. As shown earlier, with the advent of MRS, this approach has been abandoned for non-wh-expressions. I will argue that it might be wise to abandon this approach for determining the scope of wh-expressions as well, as the straightforward FRAMES inheritance approach is satisfactorily analogous to Cooper's storage technique. This will allow STORE and related features to be completely retired, while successfully handling a wider range of data. It is worth looking at Sag's (2012: 167) treatment of clausal interrogative constructions in detail. 
(17) Non-subject wh-interrogative construction:

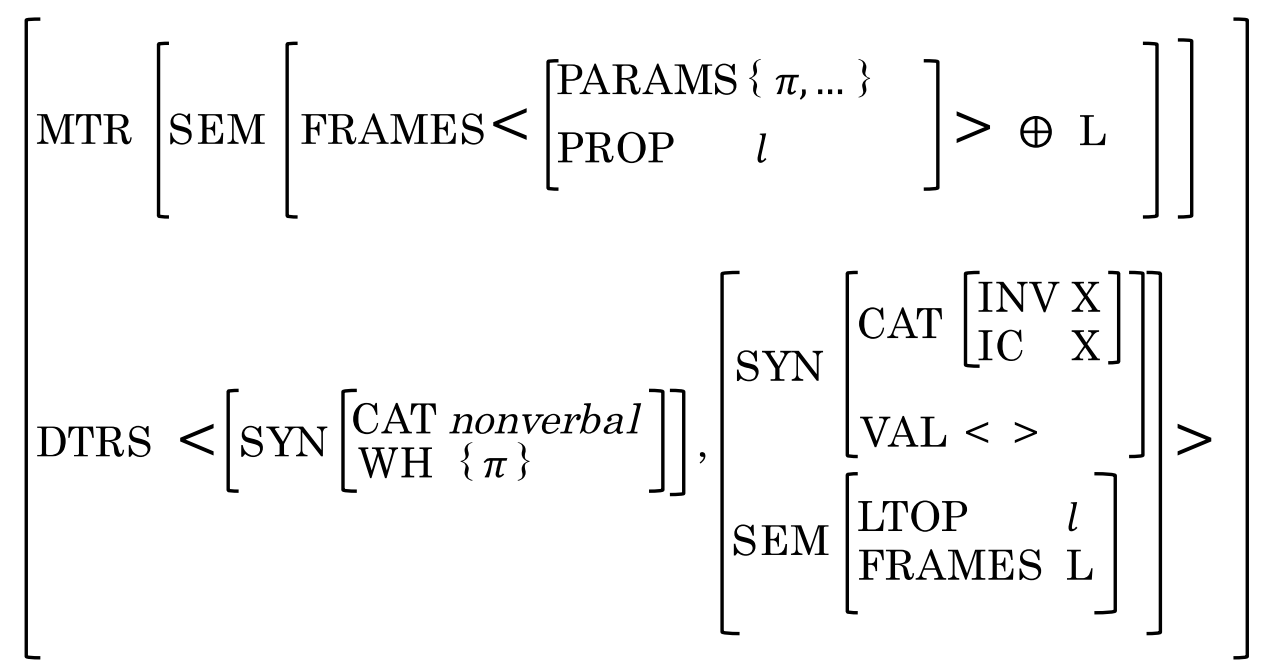

Of particular interest is the constraint whereby the FRAMES list of MTR is idiosyncratically augmented by a question-fr that introduces a parameter (PARAMS). It will be seen from (20) below that this PARAMS value is also, in fact, an appropriately bound FRAMES value. The question-fr taking wide scope at this level (via identification of PROP with the LTOP value of the sentential DTR) indicates that this is an interrogative construction. The PARAMS value appears to originate somewhat anti-intuitively in SYN of the non-head daughter. However, consider Sag's treatment of wh-words in SBCG, shown in (18):

(18) who in SBCG:

$$
\left[\begin{array}{l}
\text { FORM }<\text { who }> \\
\text { SYN }\left[\begin{array}{l}
\text { CAT }[\text { noun }] \\
\text { WH } \quad \Sigma:\{([x, \text { pers }-f r(x)])\} \\
\text { REL }\{\}
\end{array}\right] \\
\text { STORE } \Sigma \\
\text { SEM }\left[\begin{array}{l}
\text { INDEX } x \\
\text { FRAMES }<>
\end{array}\right]
\end{array}\right]
$$

From the specifications for who in (18), one can see that the PARAMS value actually originates in the syntactico-semantic interface STORE value of a $w h$-word. The WH value, structure-shared with both STORE and PARAMS, may be null (hence brackets) in case a $w h$-expression is in situ and therefore not required to trigger a wh-clause. Inheritance of STORE values is expected to be parallel to amalgamation of FRAMES. As PARAMS resolves to FRAMES in any case, if a $w h$-word such as who carries its own question-fr and a person- $f r$, this would allow for a unified MRS treatment of scope for $w h$ - and other quantificational expressions and allow STORE 
and PARAMS to be retired altogether. One might reasonably object that Purver and Ginzburg (2003) characterize CONTENT as propositional information. However, (20) below shows that Sag (2012) allows question$f r$ in MRS in any case. Perhaps question-fr is actually a special case of propositional information better characterized as required-info-fr. While more work needs to be done on the nature of FRAMES, it will be assumed here that there is no clear need for a special feature to determine scope arrangements for $w$ h-expressions. Bearing this in mind, consider (19):

(19) Who do you like?

According to Sag (2012), the resolved MRS expressions for (19) will look like (20) below. As can be seen, a question FRAME is invoked and interacts with the FRAME contributed by the verb via a PARAMS feature that idiosyncratically projects a FRAME within a FRAME.

(20) MRS resolution for (19):

$$
<\left[\begin{array}{ll}
\text { question }-f r \\
\text { PARAMS } & \left\{\left[x, \text { person }-f_{r}(x)\right]\right\} \\
\text { PROP } & l
\end{array}\right],\left[\begin{array}{ll}
\text { like-fr } & \\
\text { LABEL } & l \\
\text { EXPRNCR } & \text { you } \\
\text { UNDGR } & x
\end{array}\right]>
$$

Consider a simple alternative to (19) such as (21).

(21) Which person do you like?

For a wh-NP such as which person, Sag's (2012) approach seems to require that N' person will not contribute its FRAMES as usual, but the appropriate FRAMES value will appear in modified form in the STORE of the NP phrase. In other words, in case N' takes a wh-determiner, it unexpectedly fails to contribute its normal FRAMES value and instead projects a STORE value that is essentially identical to its FRAMES value. Furthermore, it is unclear how the wh-determiner makes its semantic contribution.

A simpler possibility is to follow the normal treatment of NPs sketched earlier, so that a wh-NP such as which person operates like other quantificational determiners and naturally contributes the question-fr that appears unexpectedly by constraint in (18). Generalizing scope assignment conditions from those applying to non-wh quantifiers (Sag 2012: 93) one would expect the question-fr to have its RESTR identified with the LABEL of N' (for example, as shown in (8) above) and its SCOPE identified with an appropriate propositional label, perhaps as in (22) below. Alternatively, again following scope assignment for quantifiers, it could be assigned wide scope via the clausal SEM|LTOP feature, parallel to Sag's PARAMS treatment. Thus question-fr would carry restrictionbearing indices (Ginsburg \& Sag 2000: 121) and would be able to take 
scope at an appropriate propositional level of structure. In this case, MRS resolution for both (19) and (21) might well be something like that indicated below. Bear in mind that SEM of the interrogative filler-head construction would also carry features for INDEX and LTOP.

(22) FRAMES in an MRS resolution for (19) and (21):

$$
<\left[\begin{array}{ll}
\text { person-fr } \\
\text { LABEL } & l_{0} \\
\text { ENTITY } & x
\end{array}\right],\left[\begin{array}{ll}
\text { question-fr } & \\
\text { LABEL } & l_{1} \\
\text { BV } & x \\
\text { RESTR } & l_{0} \\
\text { SCOPE } & l
\end{array}\right],\left[\begin{array}{lc}
\text { like-fr } \\
\text { LABEL } & l \\
\text { EXPRNCR } & \text { you } \\
\text { UNDGR } & \boldsymbol{x}
\end{array}\right]>
$$

The question-fr contributed by the non-head daughter may then be expected to take scope in accordance with the conditions applying to nonwh quantifiers.

(23) wh-interrogative construction:

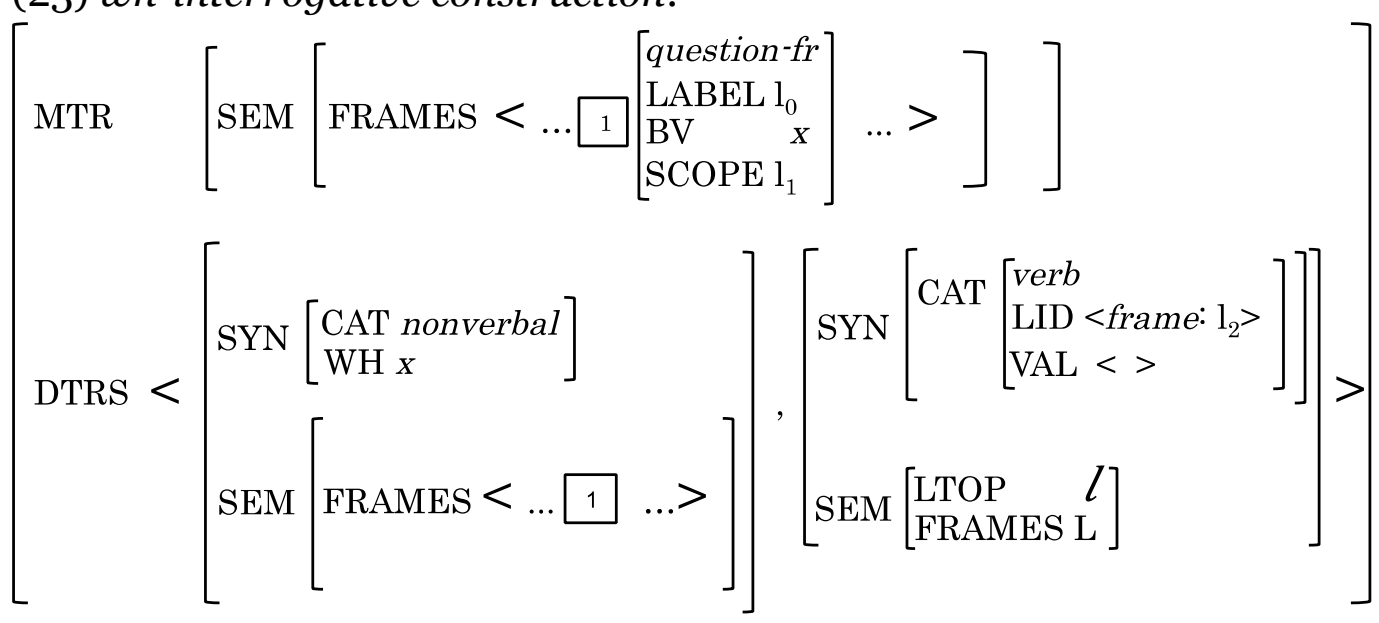

However, we propose a wh-interrogative construction, sketched in (23). This requires $w h$-interrogative constructions to have at least one questionfr. This must be amalgamated from a question-fr in SEM of the non-head daughter. No special conditions on scope assignment will be required. As for other quantificational expressions, the LABEL value of this frame may be identified with the LTOP $\left(l=l_{o}\right)$. Otherwise the SCOPE value of this question-fr is identified with LABEL of the lexical-identifier frame $\left(l_{1}=l_{2}\right)$. The LID is the frame specifying the canonical meaning of the main verb in the head daughter so such an arrangement will ensure that the whexpression takes scope at this clausal level, either wide or otherwise, entirely in line with the SBCG treatment of other quantificational expressions. The non-head daughter in turn bears a bare indexical WH value shared with the BV value of the specified question-fr. We stipulate that a question-fr will only be able to take scope at the level of whinterrogative construction. In this case, a question-fr contributed by an in- 
situ wh may, but need not, take scope in SEM of any given $w h$ interrogative construction.

\subsection{The WH syntactic trigger feature}

In (23), we make the minimalist assumption that $\mathrm{WH}$ is identical to the bound variable in the question-frame. Assuming that WH will be subject to a restricted amalgamation/inheritance condition, we may straightforwardly account for the inheritance of this syntactic trigger feature in English. We propose that certain heads (non-verbal in English) amalgamate the $\mathrm{WH}$ values from their VALENCY list and that $\mathrm{WH}$ is inherited from head daughters.

(24) *You bought [which book]?

(24) will be ruled out by the requirement for $\mathrm{WH}$ on the non-head daughter. We also assume that the verb does not amalgamate WH from its complement.

(25) *[Every worker from which department] did you fire?

Although we expect the (non-verbal) prepositional head to amalgamate $\mathrm{WH}$, (25) will be ruled out because $\mathrm{WH}$ will not be inherited from the nonhead prepositional clause daughter in the complex NP.

(26) [In which book] did you write your name?

We assume that (26) is grammatical as the (non-verbal) preposition may amalgamate $\mathrm{WH}$ from its complement and that this will be inherited from the head preposition in the $w h-P P$.

(27) [Which boy's mother's bicycle] did you paint?

(27) will also have the $\mathrm{WH}$ amalgamated from the wh-expression in the VALENCY list of the N' boy and inherited from this head onto the NP which boy. The non-verbal determiner 's is also expected to amalgamate WH from which boy as it appears in its VALENCY list, so WH will be inherited onto the DP which boy's. The same pattern of amalgamation/inheritance will be repeated to ensure that $\mathrm{WH}$ is inherited onto the $w h$-filler expression.

(28) What did everyone buy?

In (28), interpreted in light of (23), we expect the wh-expression to take either wide or narrow scope with regard to everyone. Thus an acceptable answer might correspond to a single item that everyone bought $\left(l=l_{0}\right)$ or a list of items paired with individual buyers $\left(l_{1}=l_{2}\right)$. The unified treatment suggested above, with question-frames interacting with other 
quantificational frames, successfully accounts for this ambiguity. Sag's non-subject wh-interrogative construction in (17) entirely fails to do so.

\section{Conclusion}

Sag et al (2012: 2) suggest that researchers in construction grammar are generally united by a love for complex data and hostility toward Chomskyan linguistics. The desire for minimalist solutions does not always sit well with such preferences. Kobele (2005: 391) strongly implies that the complexity of HPSG's attribute value matrices means that such theories, while valuable, are not consistent with a minimalist account of movement and feature percolation. This is regrettable, as movement and feature percolation are handled perfectly naturally in terms of inheritance (and amalgamation) conditions in unification-based accounts of the sort sketched above. Indeed, Michaelis (2012: 32) accepts that cognitive accounts of linguistic phenomena are often not taken seriously by a wider public. It is suggested here that failure to simplify contributes to this prejudice. A unified account of quantificational and wh scope conditions is not only available but also offers a better coverage of the relevant data.

\section{References}

Alqurashi, A., Borsley, R. D. 2012. Arabic relative clauses in HPSG. Proceedings of the $19^{\text {th }}$ International Conference on Head-Driven Phrase Structure Grammar. CSLI Publications, pp. 26-44.

Boas, H.C., \& Sag, I.A. (Eds.). 2012. Sign-based construction grammar. Stanford University Center for the Study of Language and Information.

Chomsky, N. 1993. A minimalist program for linguistic theory, MIT occasional papers in linguistics. Cambridge, MA: Distributed by MIT Working Papers in Linguistics. pp. 26-44.

Cooper, R. 1975. Montague's semantic theory and transformational syntax. Doctoral dissertation, University of Edinburgh.

Cooper, R. 1983. Quantification and syntactic theory. Dordrecht: Reidel.

Copestake, A., Flickinger, D., Sag, I. A., Pollard, C. 2005. Minimal recursion semantics: An introduction, Research on Language and Computation, vol. 3, pp. 281-332.

Croft, W. 2000. Explaining language change: An evolutionary approach. Harlow, Essex: Longman.

Fillmore, C. J. 1982. Frame semantics. In: Linguistics in the Morning Calm, Seoul: Hanshin Publishing Co. pp. 111-137.

Fillmore, C.J. 1985. Frames and the semantics of understanding, Quaderni di Semantica, vol. 6, pp. 222-354.

Fillmore, C. J., Baker, C. 2010. A frames approach to semantic analysis. In: B. Heine and H. Narrog (Eds.) The Oxford Handbook of Linguistic Analysis. Oxford: Oxford University Press. pp. 313-340

Fillmore, C. J., Kay, P. 1993. Construction grammar coursebook. Berkeley, Calif.: Copy Central.

Ginsburg, J., Sag, I.A. 2000. Interrogative investigations: The form, meaning, and use of English interrogatives. CSLI Publications. 
Kobele, G.M. 2005. Features moving madly: A formal perspective on feature percolation in the minimalist program. Research in Language and Computation, vol. 3, pp. 391-410.

Michaelis, L. A. 2012. Making the case for construction grammar. In: Boas, H.C., Sag, I.A. (Eds.). Sign-based construction grammar. Stanford University Center for the Study of Language and Information. pp.31-67.

Pollard, C., Sag, I.A. 1994. Head-Driven Phrase Structure Grammar. Chicago.

Pollard, C., Yoo, E.J. 1998. A unified theory of scope for quantifiers and whphrases. Journal of Linguistics, vol. 34, pp. 415-445.

Purver, M., Ginzburg, J. 2003. Clarifying noun phrase semantics in HPSG. Proceedings of the $10^{\text {th }}$ International Conference on Head-Driven Phrase Structure Grammar. CSLI Publications, pp. 338-358.

Sag, I.A. 1997. English relative clause constructions, Journal of Linguistics, vol. 33, pp. 431-484.

Sag, I.A. 2012. Sign-based Construction Grammar: An informal synopsis. In: Boas, H.C., Sag, I.A. (Eds.). Sign-based construction grammar. Stanford University Center for the Study of Language and Information. pp. 69-202.

Tomasello, M. 2003. Constructing a language: A usage-based theory of language acquisition. Boston, MA: Harvard University Press.

Yoo, E-J. 2002. A lexical approach to English floating quantifiers. Berkeley Linguistics Society, vol. 21, pp. 351-362. 